\title{
Hydroelastic Buffeting assessment over a Vertically Hinged Flat Plate
}

\author{
Mohammadmehdi Armandei ${ }^{*(1)}$, Antonio Carlos Fernandes ${ }^{(2)}$, Ali Bakhshandeh Rostami ${ }^{(3)}$ \\ (1) Post-doctoral fellow, (2) Associate professor, (3) PhD student. \\ COPPE, Federal University of Rio de Janeiro, RJ, Brazil.
}

\begin{abstract}
This study explores hydroelastic buffeting of a flat plate experimentally. The flat plate is hinged vertically to an elastic axis, and its only degree of freedom therefore is rotation in pure yaw about this axis. Buffeting is a type of flow-induced oscillation which is caused by unsteadiness in the incoming flow. The laboratory technique presented here was applied for buffeting stimulation of the flat plate in water current. A bluff body was placed in the upstream of the flat plate to impose the unsteadiness. During the experiments, the distance between the bluff body and the flat plate was changed several times to assess the effect of distance on buffeting behavior. According to results, for distance ratios greater than 1 , the buffeting responses are independent of distance ratio, although they are dependent on Reynolds number. Also, in order to show the feasibility of hydrokinetic energy harvesting through buffeting is discussed.
\end{abstract}

Keyword: hydroelastic buffeting, buffeting stimulation, flow-induced oscillation, hydrokinetic energy harvesting.

\section{1- Introduction}

Aeroelasticity is a branch of engineering that studies the aerodynamic interaction between wind and elastic structures. Flow-induced oscillation is the most common outcome of this interation,

* Corresponding author. Email: marmandei@oceanica.ufrj.br 
which occurs in different engineering disciplines such as hydrodynamic. In these cases, the theory of hydroelasticity, which is adapted from aeroelasticity, is applied to describe the effect of structural response of the body on the fluid around it. An oscillator which is influenced by hydrodynamic forces can be called a hydroelastic oscillator. In contrast to the steady hydrodynamics of a structure where only the shape and relative orientation to the current field is relevant, in hydroelasticity the influence of the hydrodynamic forces on the structural motion has to be taken into account.

Regarding to hydrodynamic unsteadiness, different types of flow-induced oscillations can be distinguished as a basically resonance or instability type [1]. In resonance type, the unsteadiness of the forces is independent of the structural motion and an oscillatory forcing is applied even for a stationary structure. The external forces are generated by turbulence, gust and vortices in the free-stream. Moreover, sustained oscillations will occur if the oscillatory forcing has a significant component with a frequency corresponding to one of the natural frequencies of the structure. On the other hand, for instability type, the motion of the structure is a consequence of the flowinduced forces created by the shed wake alone. Indeed, the hydrodynamic forces vary with time only as a result of the motion of the structure itself. These motion-induced forces can either decrease or increase the oscillation amplitude.

According to the classification above (summarized in Figure 1), buffeting and vortex-induced vibration are categorized as resonance type. Buffeting is caused by the unsteadiness of the incoming flow, which may be due to natural turbulence, or by the presence of the wake of upstream objects. Vortex-induced vibration is caused under the influence of the alternate vortex shedding from the rear of bluff bodies [1]. On the other side, flutter and galloping will be classified as instability type [2]. 
The investigations of Gopalkrishnan et al. [3] and Streitlien et al. [4] are examples of resonance type flow-induced oscillation. They considered the motion of an oscillating airfoil in a uniform stream with a row of vortices superimposed on it. The airfoil oscillations are externally imposed. In both studies, the oscillations of the airfoil generated by flow-induced forces, whether they are created by the oncoming vortices or by the induced motion of the shed wake, are not considered. As a result, the important parameter is the phase between the mechanically induced airfoil motion and the arrival of the free-stream vortices. Furthermore, Beal et al [5] worked on passive propulsion mode of high aspect ratio foil which is influenced by oncoming vortices of upstream bluff body. Their investigated problem was two degree of freedom. Although, their mechanical system was proposed to probe into the fish propulsion mechanism, they have inquired into the potential of energy extraction by this system. Eventually, mechanical energy had been extracted from the flow at the same time that thrust was produced.

Moreover, Allen \& Smits [6] showed that a piezoelectric membrane subject to the action of oncoming vortices from a bluff body can extract energy from the flow. Streitlien et al. [4] showed theoretically that a foil can extract energy from oncoming vortices resulting in propulsive efficiency higher than $100 \%$.

Additionally, So et al. [7] examined numerically a simple fluid-structure interaction problem, which is represented by the unsteady response of an airfoil/blade to a Karman vortex street in an inviscid uniform flow. Two different cases were examined; one with a rigid airfoil/blade, where the structural stiffness is infinite, another with an elastic blade. A time-marching technique solving the Euler equations and a two-degree-of-freedom structural dynamic model was used to examine the interactions between the fluid and the structure. 
This paper will present the laboratory technique applied for buffeting stimulation of the flat plate in water current. This problem is one degree of freedom. The flat plate is hinged vertically and can rotate around its elastic axis (as yaw motion). Four linear springs are mounted on the plate to provide the elasticity. A D-section cylinder is applied as a bluff body in the upstream of the flat plate with a diameter equal to a half of the flat plate's chord length.

An alternative energy solution for small, remote sensing applications which has received considerable attention recently is energy harvesting through flutter [refrence! (cite me)]. Therefore, to demonstrate the applicability of buffeting in energy harvesting, a mechanical system is proposed through this work to convert the oscillating motion into the rotation. The output rotation can be applied to do some measureable physical work such as lifting a weight, to assess the energy harvesting performance.

\section{2- Experimental tests}

\section{2-1- Instrumentation}

All of the experimental tests were conducted in the current flume of LOC (Laboratory of Waves and Currents) in COPPE/UFRJ. The current flume has $22 \mathrm{~m}$ length, $1.4 \mathrm{~m}$ width and $0.5 \mathrm{~m}$ depth, and the maximum current speed attainable in the flume is $0.5 \mathrm{~m} / \mathrm{s}$. The free stream current speed was recorded using an electronic flow meter.

A set-up was prepared consisting of an aluminum made $\left(\rho_{A l}=2700 \mathrm{Kg} / \mathrm{m}^{3}\right)$ rectangular flat plate with $0.6 \mathrm{~m}$ height $(h)$ and $0.3 \mathrm{~m}$ chord length $(c)$, and an elastic axis along its chord length. Therefore, the flat plate has only one degree of freedom which is rotation in pure yaw about its elastic axis. The yaw motion was captured via a Qualisys motion capture system (2 Oqus cameras and the QTM software version 1.1). In order to provide the elasticity, it was connected to 4 identical linear springs, each in one of its 4 edges. The current flume layout with the 
arrangement of the instruments and the schematic top view of the set-up are shown through Figure 2.

Basically, the uncertainties in the measurements come from three sources; experimental set-up, the data acquisition devices, and truncation error that are taken into account in numerical analysis. In order to check the former, each test was performed several times during the experiments, to assure the repeatability. The data acquisition devices also were configured to reduce the uncertainties as much as possible. For instance, Qualisys motion capture system was set at 100 frames per second to capture the displacement with more clarity. Furthermore, calibration tests on the electronic flow meter demonstrated that its data acquisition precision is high, with about $1 \%$ of uncertainty [8].

\section{2-2- $\quad$ Tests description}

Using the static equilibrium equation for the system, the equivalent torsional spring rate is

$$
k_{\theta}=2 k c^{2}\left[1-2\left(\frac{a}{c}\right)+2\left(\frac{a}{c}\right)^{2}\right]
$$

where $k$ is the linear spring constant, and $a$ represents the distance of the elastic axis from the leading edge of the flat plate. The elastic axis is able to move along a portion of the chord length located between $0.5 c$ and $0.9 c$. Different groups of springs were utilized, each group with equal rates. All the springs were calibrated and their rates were obtained.

The total damping ratio is a very important parameter for energy extraction, which includes structural and fluid damping. The former is caused by friction at the component and the material scales, and the latter is the result of energy dissipation, as the fluid moves relative to the 
oscillating plate. Since there is no analytical estimation, the total damping ratio was found by conducting several decay tests in still water. Finally, its mean value was found to be $\zeta_{T}=0.33$.

The unsteadiness was imposed via placing a D-section cylinder of $0.15 \mathrm{~m}$ diameter $(D)$ in the upstream of the flat plate, as depicted in Figure 2. The D-section cylinder acts as a stimulator for buffeting. Thus, the stimulation frequency is

$\omega=\frac{2 \pi S t U}{D}$

where $S t$ is the Strouhal number of the vortex shedding behind the D-section cylinder. Since there is a little information in the literature about the Strouhal number of the vortex shedding behind a D-section cylinder at the existing range of Reynolds number based on $D$ (30000 $\left.\leq R e_{D} \leq 95000\right)$, some tests were conducted with the D-section cylinder alone. As it can be seen from Figure 3, the Strouhal number values are mostly close to 0.23.

The response angle $\theta$ of the flat plate in buffeting is a function of $c, U, a, d, D$, and $k_{\theta}$. A dimensional analysis of these parameters leads to

$\theta=f\left(\frac{a}{c}, \frac{d}{c}, R e_{D}, k^{*}\right)$

where $k^{*}$ is defined as

$$
k^{*}=\frac{k}{\frac{1}{2} \rho U^{2} c}
$$


In order to have a dimensionless parameter to represent only the linear spring effect, the current speed dependency should be eliminated from Equation (14). To this end, a new dimensionless parameter $(\kappa)$ is defined by multiplying $k^{*}$ by $\operatorname{Re}_{D}^{2}\left(\operatorname{Re}_{D}\right.$ squared $\_2^{\text {nd }}$ comment). It gives

$\kappa=\frac{k c}{\frac{1}{2} \rho v^{2}}$

Through the experiments, six different cases were tested, combinations of two different $\kappa$ s and three different $a / c$ ratios. Table 1 lists the tested cases. In order to see the responses, each case was tested for $30000 \leq R e_{D} \leq 75000$ and $1 \leq d / c \leq 2.67$. Then, using the captured responses the ideal hydrokinetic energy extraction performance through buffeting was estimated.

\section{3- Results}

According to the time histories captured, for high $R e_{D}$ the yaw motion is a narrowband (singlefrequency) oscillation, whereas for low $R e_{D}$ it is a broadband (noise-like) oscillation. The reason is because the oscillation frequency for low Reynolds numbers is different than the vortex shedding frequency. Figure 4 shows 2 samples of the yaw motion time histories taken from Case 3. It can be seen that they both have a sinusoidal behavior. Therefore, the frequency was calculated by taking FFT (Fast Fourier Transform) of the captured time histories. The maximum response amplitude also was found by

$\theta_{\max }=\sqrt{\frac{2 \sum_{i=1}^{N} \theta_{i}^{2}}{N}}$

Figures 5 and 6 show the response frequency and the maximum response amplitude of buffeting versus the distance ratio, for different $R e_{D} \mathrm{~S}$ and for all cases given in Table 1 . It is seen that at the 
middle the distance ratios, the buffeting responses are independent from the distance ratio and increase with $\operatorname{Re}_{D}$. Due to the yaw motion of the plate some waves are created on the surface and propagated to the upstream. For the very small distance ratios, these waves affect the von Kármán vortex street behind the D-section cylinder and hence the buffeting responses.

The largest $\theta_{\max }$ values are observed in Cases 3 and 5, Case $3\left(\theta_{\max }=0.355 \mathrm{rad}\right)$ for $R e_{D}=75000$ and Case $5\left(\theta_{\max }=0.484 \mathrm{rad}\right)$ for $R e_{D}=60000$. Figure 7 demonstrates that in both cases, the response frequency of the yawing flat plate is, as expected, very close to its natural frequency. The largest $\theta_{\max }$ obtained imply that resonance happens in cases 3 and 5 .

Through a similar study attempting for energy extraction with a membrane in the wake of a bluff body, the resonance was found to be identical to the optimal flow coupling requirement in the performance [9]. The next section is about the transmission system to be installed for hydrokinetic energy extraction.

\section{4- Transmission system description}

The current research was made as a preliminary study to explore hydroelastic buffeting. At this stage only the responses were captured and analyzed. However, by designing and installing a transmission system on top of the elastic axis one can be able to convert the angular oscillation to rotation. A transmission system was designed in a parallel study in order to convert the oscillation due to torsional galloping of the flat plate to rotation [10]. The same system can be applied here in order to assess the hydrokinetic energy extraction capability of buffeting. In order to clarify the mechanism of the transmission system, it photograph is shown in Figure 8 . The transmission box has two shafts; the input shaft which is attached to the elastic axis of the flat plate, and the output shaft which gives the rotation to a sheave. Into the box, the input shaft and 
the output shafts are connected to bicycle ratchets. The input ratchets are positioned one on top of the other and are connected to the output ratchets with a pair of chains. Also, the output ratchets are connected together with two gears on top of them. The alignment of the ratchets is so that the oscillation of the input ratchets leads to the pure rotation of the output ratchets. It is possible to use the rotation of the both output shafts to lift the weight, but here only one of them was used. The rotation in the output shaft can perform a physical work to measure the performance, for example twisting a string to lift a weight up.

\section{5- Conclusion}

A preliminary study on hydroelastic buffeting of a flat plate in the wake of a bluff body was presented through this study. The laboratory technique was discussed to stimulate the phenomenon. Six different cases were tested, combinations of two different $\kappa$ s and three different $a / c$ ratios. On the basis of the experiment results for the distance ratios larger than 1 , the buffeting responses are independent of the distance ratio, although increase with Reynolds number based on the bluff body diameter $\left(R e_{D}\right)$. Also, for some experimented cases, resonance phenomenon was observed wherein the largest $\theta_{\max }$ are obtained. The resonance can be the optimal flow coupling for the sake of hydrokinetic energy extraction. Therefore, to demonstrate the applicability of buffeting in energy harvesting, a mechanical transmission system was proposed to convert the oscillating motion into rotation in order to lifting weight.

\section{Acknowledgment}

The authors express their thanks to $\mathrm{CNPq}$ (the Brazilian National research Council), ANP (Brazilian National Petroleum Agency), and LOC/COPPE/UFRJ (Laboratory of Waves and Current of COPPE, Federal University of Rio de Janeiro).

\section{Reference}


[1] Van Oudheusden B.W., 1992, "Investigation of an Aeroelastic Oscillator: Analysis of One-Degree- ofFreedom Galloping with combined Translational and Torsional Effects", Report LR 707, Delft University of Technology

[2] Bisplinghoff R. L., Ashley H., Halfman R.L., 1955,” Aeroelasticity “, New York, Dover.

[3] Gopalkrishnan R., Triantafyllou M. S., Triantafyllou G. S., Barrett D. 1994 “Active vorticity control in a shear flow using a flapping foil”, Journal of Fluid Mechanics, 274, 1-21.

[4] Streitlien K., Triantafyllou G. S., Triantafyllou M. S. 1996 "Efficient foil propulsion through vortex control”, AIAA Journal 34, 2315-2319.

[5] Beal D.N., Hover F.S., Triantafyllou M. S., Triantafyllou M. S., Liao J.C., Lauder G.V., 2006, "Passive Propulsion in Vortex Wakes", Journal of Fluid Mechanics 549, 385-402

[6] Allen, J. J. \& Smits, J. 2001 “Energy harvesting eel” J. Fluids Structure, 15, 629-640.

[7] So, R. M. C., Jadic, I. \& Mignolet, M. P. 1999 Fluid-structure resonance produced by oncoming alternating vortices. J. Fluids Struct. 13, 519-548.

[8] Fernandes A.C., Bakhshandeh Rostami A., Canzian L.G., Mirzaei Sefat S. "Vertical Axis Current Turbine (VACT) and its Efficiency”, ASME 2013 32nd International Conference on Ocean, Offshore and Arctic Engineering (OMAE2013), Nantes, France, June 9-14, 2013

[9] Allan, J. J., Smits, A. J., 2001, “Energy harvesting eel”, Journal of Fluid and Structures, 15, $629-640$.

[10] Armandei, M., Fernandes, A. C. "Marine Current Energy Using Torsional Galloping Based Turbine”. Offshore Technology Conference. Houston. TX. USA. 2013. 


\section{List of Figure Captions}

Figure 1 - Flow-induced oscillation flowchart

Figure 2 - (a) Current flume layout with the arrangement of the instruments, (b) Schematic top view

Figure 3- Vortex shedding Strouhal number behind the cylinder versus the Reynolds number

Figure 4- (a) Narrowband (single-frequency) oscillation, (b) Broadband (noise-like) oscillation. Time histories are taken from Case 3.

Figure 5- The response frequency versus the distance ratio for all cases given in Table 1.

Figure 6- The maximum response amplitude versus the distance ratio for cases given in Table 1.

Figure 7- Efficiency versus the frequency ratio $\left(\omega / \omega_{n}\right)$ for the different distance ratios (including case 1 !)

Figure 8- transmission system photograph 


\section{Tables}

Table 1- The combinations for each case

\begin{tabular}{ccc}
\hline Case number & $a / c$ & $\kappa\left(\times 10^{10}\right)$ \\
\hline 1 & 0.5 & 3.615 \\
2 & 0.5 & 11.816 \\
3 & 0.73 & 3.615 \\
4 & 0.73 & 11.816 \\
5 & 0.9 & 3.615 \\
6 & 0.9 & 11.816 \\
\hline
\end{tabular}




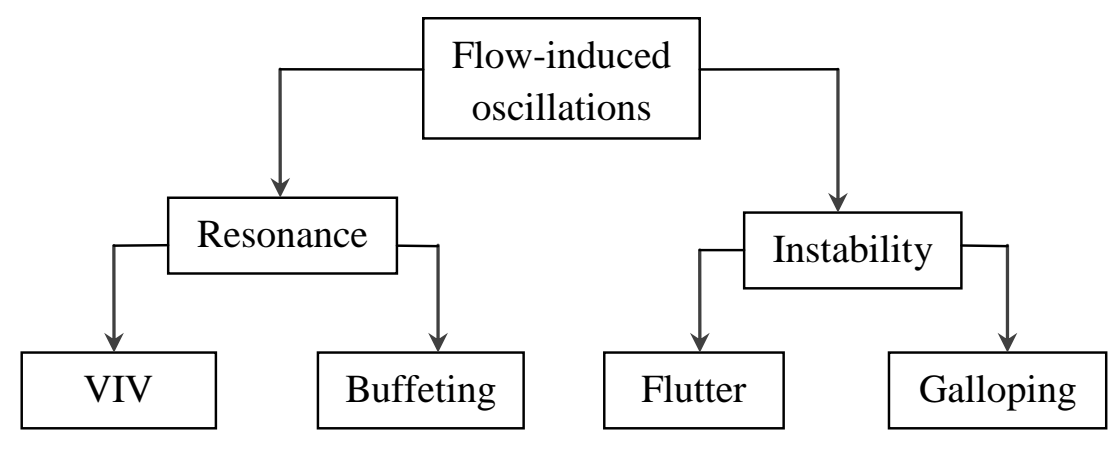



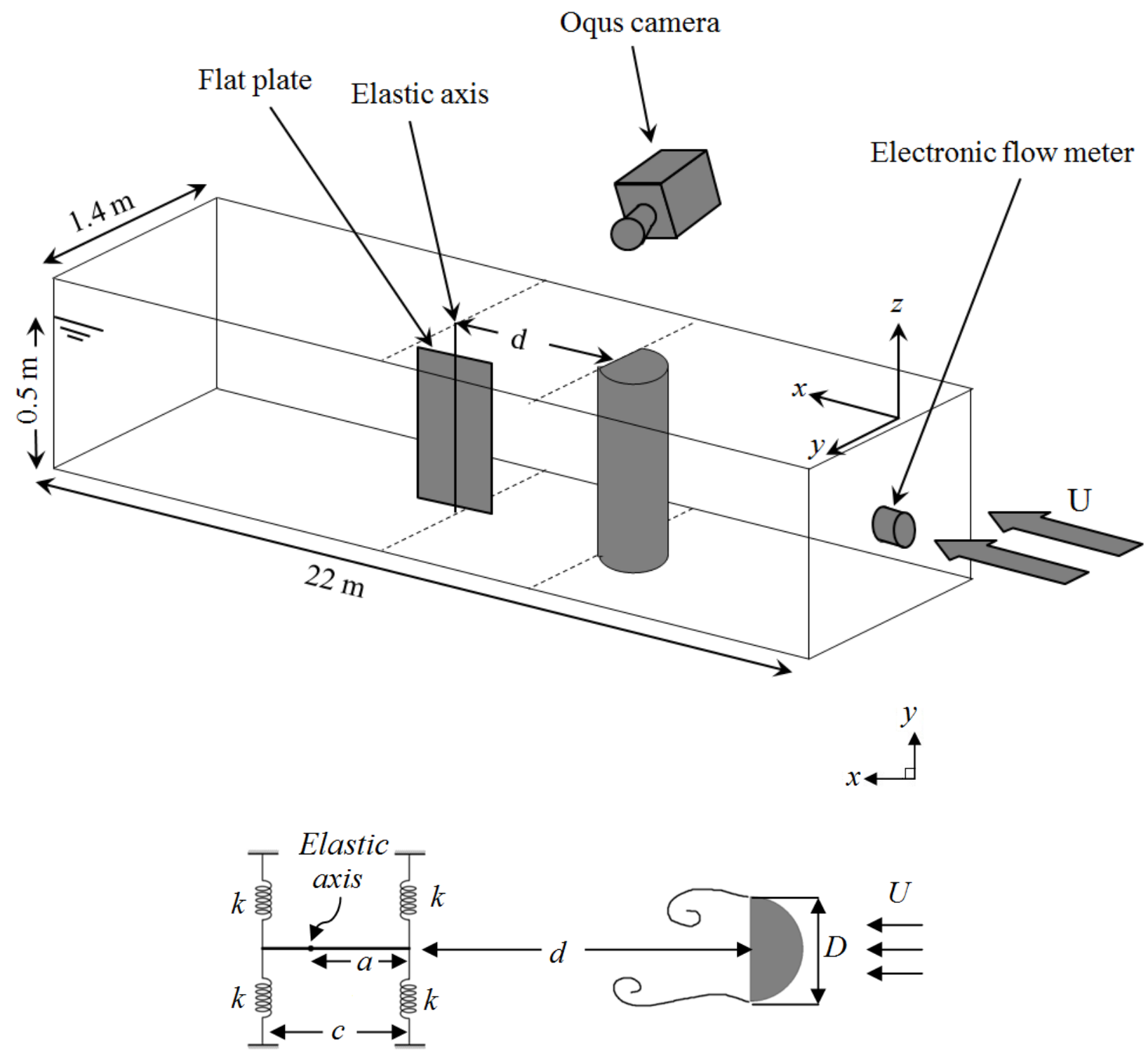


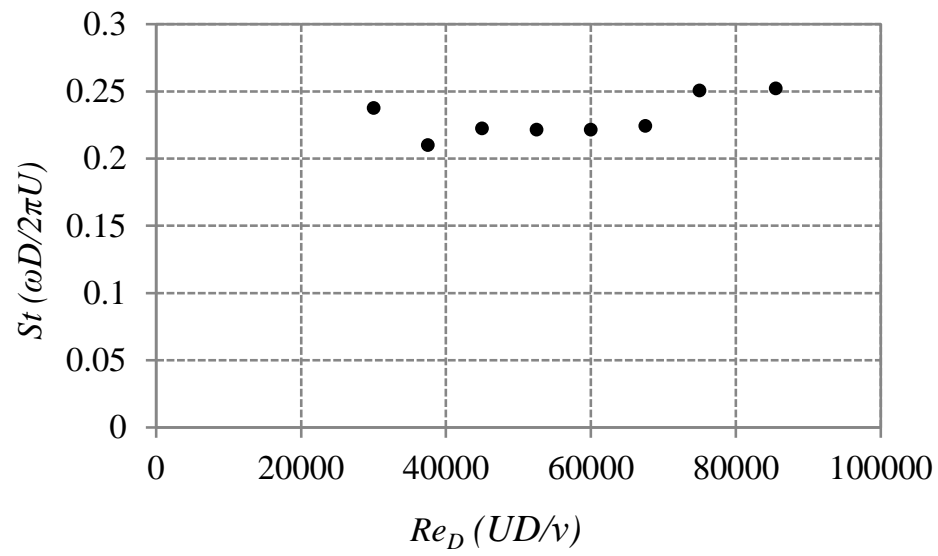



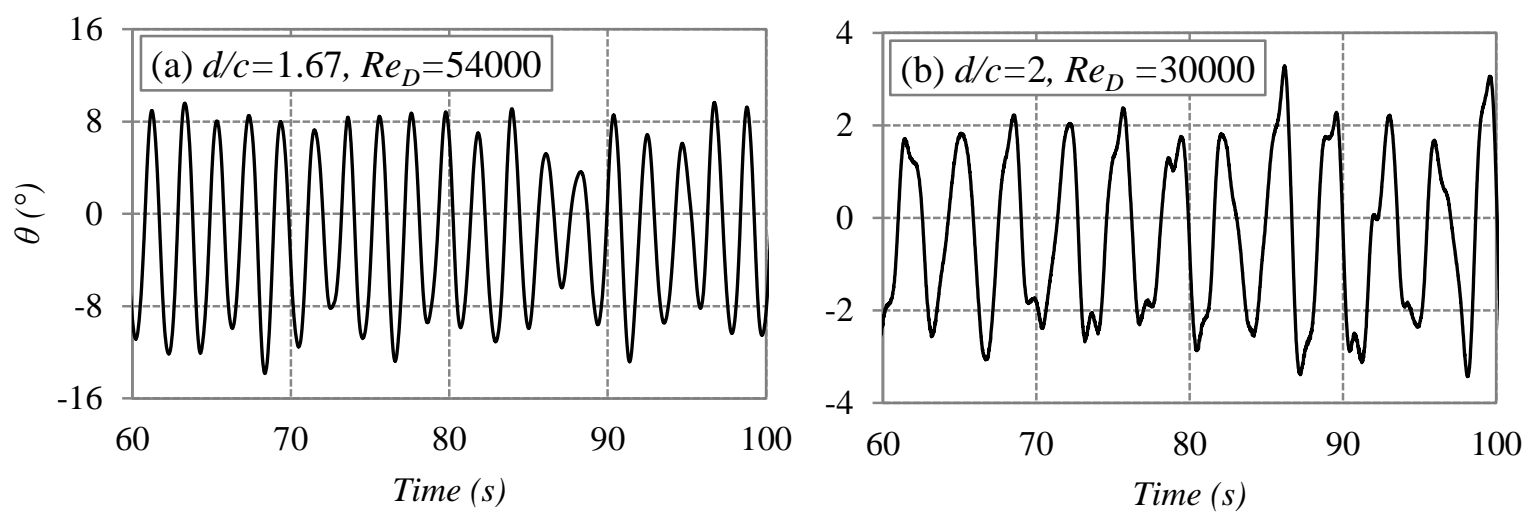

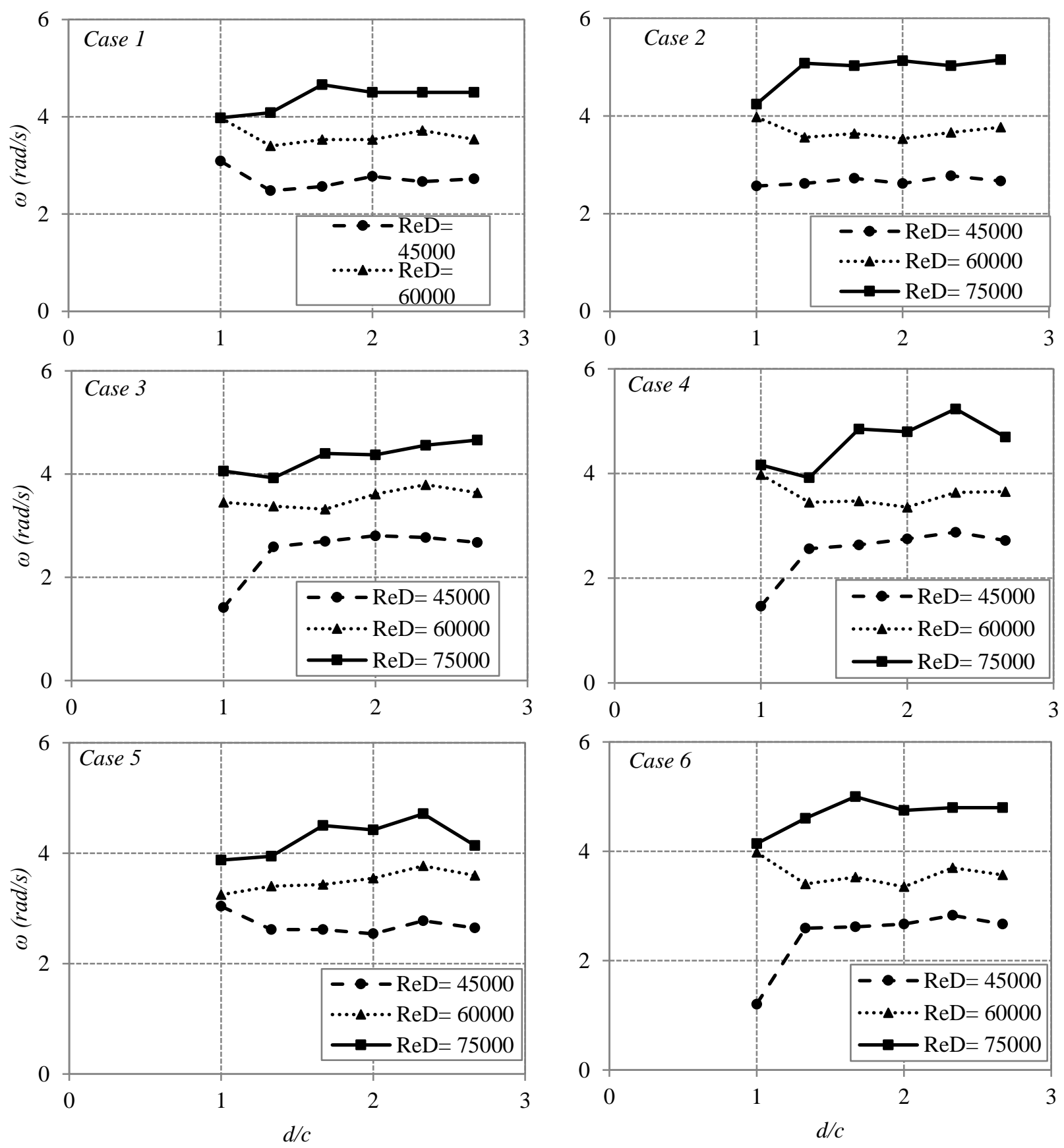

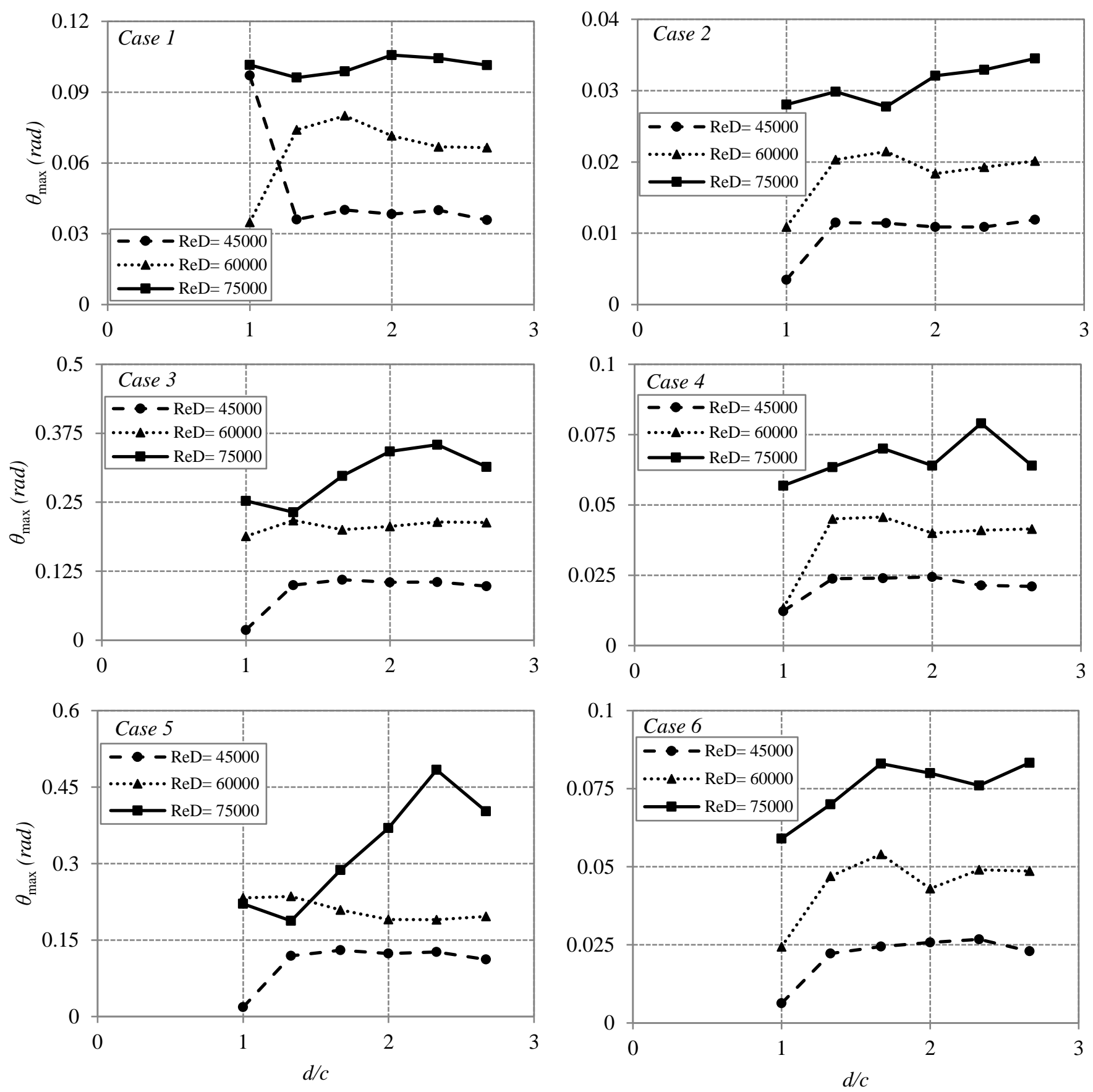

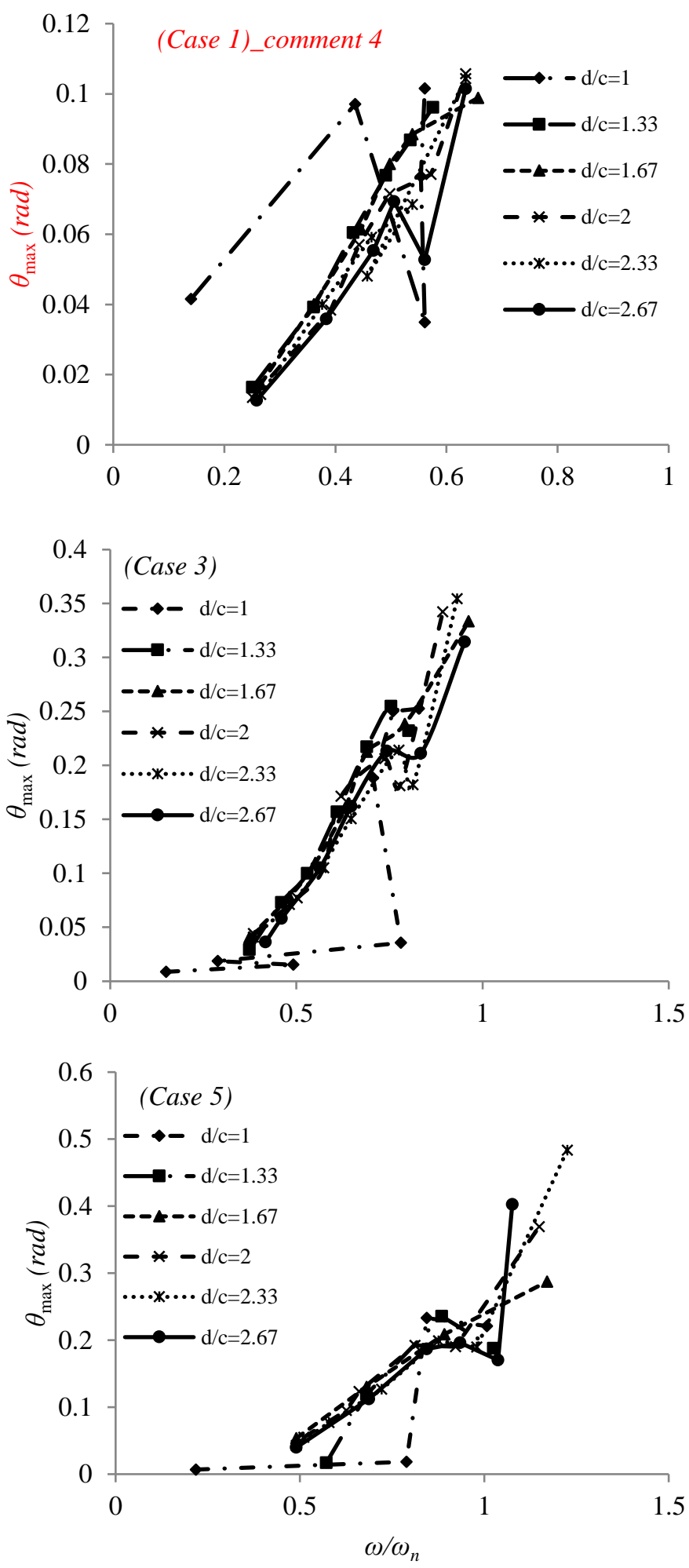


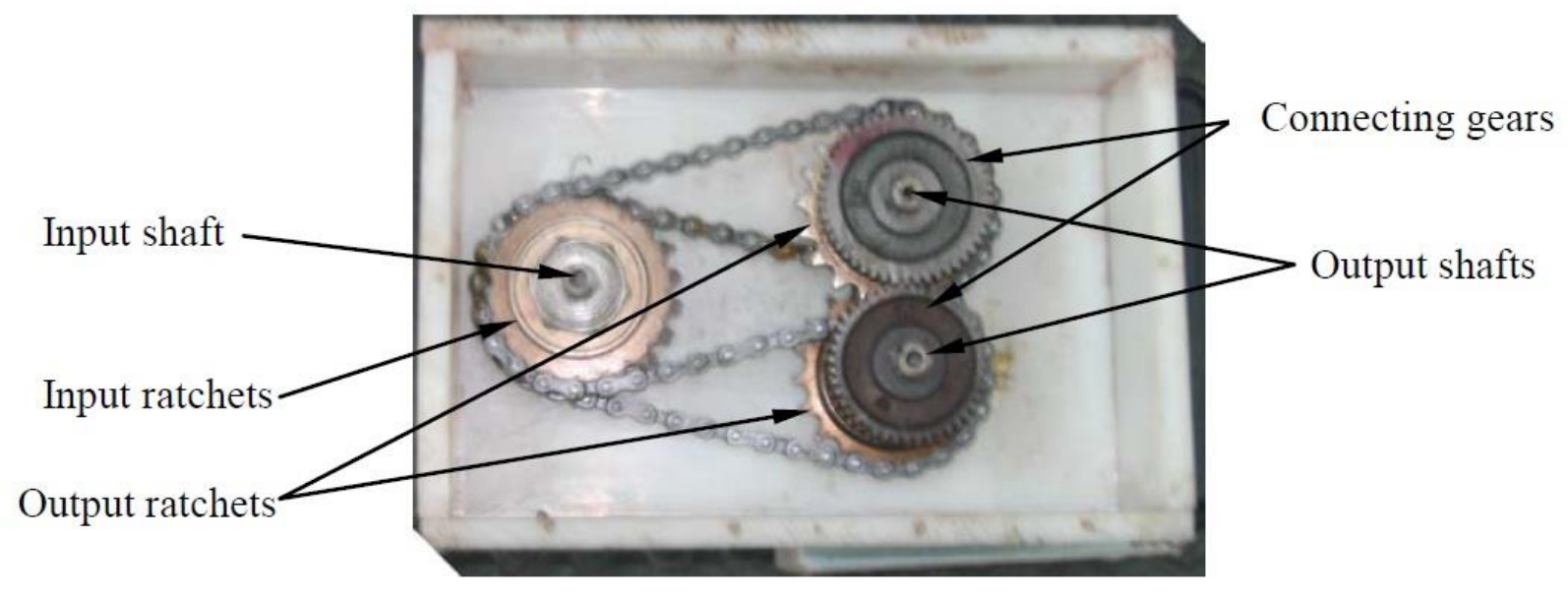

